\title{
PLANETARY EPHEMERIDES
}

\author{
R. L. DUNCOMBE, P. K. SEIDELMANN, and P. M. JANICZEK \\ U.S. Naval Observatory, Washington, D.C., U.S.A.
}

At the present time the planetary ephemerides in the Astronomical Ephemeris and in the American Ephemeris and Nautical Almanac (both hereinafter referred to as the AE), the Astronomical Ephemeris of the U.S.S.R. and most other national almanacs have the following basis: For Mercury, Venus, Earth, and Mars the general theories of Simon Newcomb (1898a), the ephemeris of Mars including the empirical corrections determined by Ross (1917); for the five outer planets, the numerical integration of Eckert et al. (1951); the Connaissance de Temps publishes ephemerides of Mercury, Venus, Earth, and Mars based on the theories of Leverrier (1858, 1859, 1861a, b); for Jupiter, Saturn, Uranus, and Neptune the ephemerides are based on Leverrier's (1876a, b, 1877a, b) expressions as modified by Gaillot $(1904,1910,1913)$. The ephemeris of Pluto is based on the numerical integration of Eckert et al. In all of the above publications the ephemeris of the Moon is now based on the Improved Lunar Ephemeris which is derived from the theory of Brown (1919). Newcomb's theories and the numerical integration of the orbits of the five outer planets all rest primarily on the system of astronomical constants and planetary masses adopted at the Paris conferences of 1896 and 1911 (Monthly Notices Roy. Astron. Soc., 1912).

With the passage of time these basic theories and their underlying constants have been found to be defective in numerous respects. Analyses of extended series of precise meridian circle observations, as well as the advent of radar and laser observing techniques and the use of space probes, have permitted the determination of more precise values of many of the basic astronomical constants. The availability of these new data precipitated the Paris Symposium of 1963 on the System of Astronomical Constants. The results of this Conference were formally adopted by the 13th General Assembly in 1964 as the IAU System of Astronomical Constants, and commencing in 1968 the effects of the new system were incorporated into the national ephemerides (Supplement to AE 1968). Several constants remained unchanged in the new system, however, and it was recommended that they be considered later. Precession and the masses of the principal planets are among them. As a consequence of discussions at IAU Colloquium No. 9, Heidelberg 1970, and in accordance with the recommendations of Commission 4 of the IAU, the 14th General Assembly set up working groups to study the subjects of Precessional Constants, Units and Time Scales, and Planetary Ephemerides. Commission 4 has requested that the Working Group on Planetary Ephemerides consider the system of planetary masses to be used in a new set of fundamental"ephemerides as well as other factors that it considers to be relevant to the adoption of a new"set of ephemerides, including choice of orbital elements or starting values, coordinate systems, form of equations and precision in computation.

The membership comprises R. Duncombe, convener; P. Janiczek, secretary; 
J. Kovalevsky, V. Abalakin, D. O'Handley, C. Oesterwinter, B. Morando, A. Sinclair, J. Schubart, W. Klepczynski, and S. Herrick. Through correspondence there has been an exchange of opinions among members of the Working Group and, in addition, opinions and recommendations have been solicited from the following consultants: L. Carpenter, G. M. Clemence, A. Deprit, L. E. Doggett, D. Dunham, A. D. Fiala, J. Griffith, P. Herget, H. Hertz, R. Laubscher, J. H. Lieske, B. Marsden, J. D. Mulholland, D. Pascu, P. K. Seidelmann, I. I. Shapiro, T. C. Van Flandern, W. Zielenbach.

Discussions and correspondence concerning the form of the fundamental theories seem inevitably to divide the participants into two groups, one defending the methods of analytic and general perturbations, the other favouring special perturbations by numerical means. It would be possible to objectively present a detailed list of the advantages and disadvantages of the two classic methods; but to do so would invite the continuation of a debate which has had, and is likely to have, no resolution that would satisfy every need and purpose. Among the national ephemeris offices charged with the responsibility of fundamental ephemerides, however, there is one practical requirement that is unavoidable. The interval of time between the adoption of new fundamental ephemerides and their publication is several years. For example, in order to introduce new ephemerides in the $1980 \mathrm{AE}$, the fundamental theories must be established and available by 1976 . Moreover, the astronomical constants which enter the ephemerides should be available when the calculations begin. This means that the time for decisions to be made is almost at hand. As a consequence of the scheduling requirements, a choice may be made to utilize the method of numerical integration, to be followed later by consistent analytic theories which would serve over a more extended period.

At this time it seems appropriate to comment on the frequently stated opinion that ephemerides will be subject to frequent revisions dictated by highly accurate observations, such as radar and laser ranging. This is currently an unrealistic assessment. In order to exploit the accuracies of ranging techniques to their fullest potential, they must be conducted within the framework of continuing and systematic observational programs. Only after extended series of observations have accumulated will it become possible to determine the various separate effects of a complex planetary system which influence observations made from within the system. It is noted that there are presently no systematic programs of planetary radar observations; and, in fact, there are very few such observations being made at all for fundamental purposes.

For the accuracy required by the published ephemerides, a newly calculated system of ephemerides may be satisfactory for a period of time. A more accurate set of ephemerides, available only in machine readable form, may change more frequently, but this would not be influenced by nor affect the restraints of publication deadlines.

In addition to the method of calculating the ephemerides and the requirement of a series of observations, the underlying constants must be specified. The system of planetary masses used in the presently published ephemerides is basically that derived 
and introduced by Newcomb $(1898 \mathrm{a}, \mathrm{b})$. The availability of extended series of meridian circle observations of the planets since the date of his analysis and the advent of radar, laser, and spacecraft data have made possible several refinements in our knowledge of the masses of the principal planets. Thus far the new observing techniques have been confined to the Moon, Mercury, Venus, and Mars; and therefore the most dramatic refinement of our knowledge would be expected with reference to these particular bodies.

A few of those consulted by the Working Group favor the adoption of a system of planetary masses arising from a single global investigation, while at the other extreme an opinion was given that the system of masses should be left flexible and unspecified. A system of planetary masses stemming from a single global investigation may in a sense be self-consistent, but it will normally not reflect all the types of data which provide information on the masses. Also, the resulting mass values are not necessarily of uniform accuracy, nor is the possibility eliminated for one value to be systematically in error. Generally speaking, the mass of a principal planet may be determined from the perturbations induced in the motion of a neighboring planet or a nearby spacecraft, or from the orbits of its natural or artificial satellites. It has been the established practice in the past to derive a system of planetary masses by the judicious combination of all the observational determinations. The majority opinion of the working group respondents favors the consideration of such a compiled system of planetary masses. Since the advent of new data techniques for the determination of planetary masses, such as spacecraft flybys and artificial satellites, two independent comprehensive discussions of all observationally determined values have been made (Kovalevsky, 1971; Duncombe et al., 1973).

The first discussion reflects the experienced scientific judgment of the investigator in rating the various observational determinations to arrive at suggested values of the masses. In the second discussion an effort was made to apply statistical techniques to the combination of all observational determinations in order to minimize the effect of systematic errors known to be present in the various observational types. Due to the paucity of observational determinations in a few cases, the statistical approach was not entirely successful; and therefore to some extent the subjective judgments of the compilers are involved in both systems.

Table I presents the currently adopted IAU values, the statistically based mean values, Kovalevsky's suggested values, and the authors' recommendations for values to be adopted in preparing new ephemerides. Also, comments are given to explain the selections. It is hoped that this list of reciprocal masses will form the basis for further discussion.

Before new ephemerides can be determined, the other Working Groups must make their recommendations. To meet the desired deadline for the new ephemerides of 1980 , it is suggested that a colloquium be held in 1974 to tentatively adopt new constants, planetary masses, the gravitational model, the fundamental equator and equinox, and other guidelines for calculating new ephemerides. Cognizant organizations could calculate ephemerides, fit them to observations and intercompare the results. The 


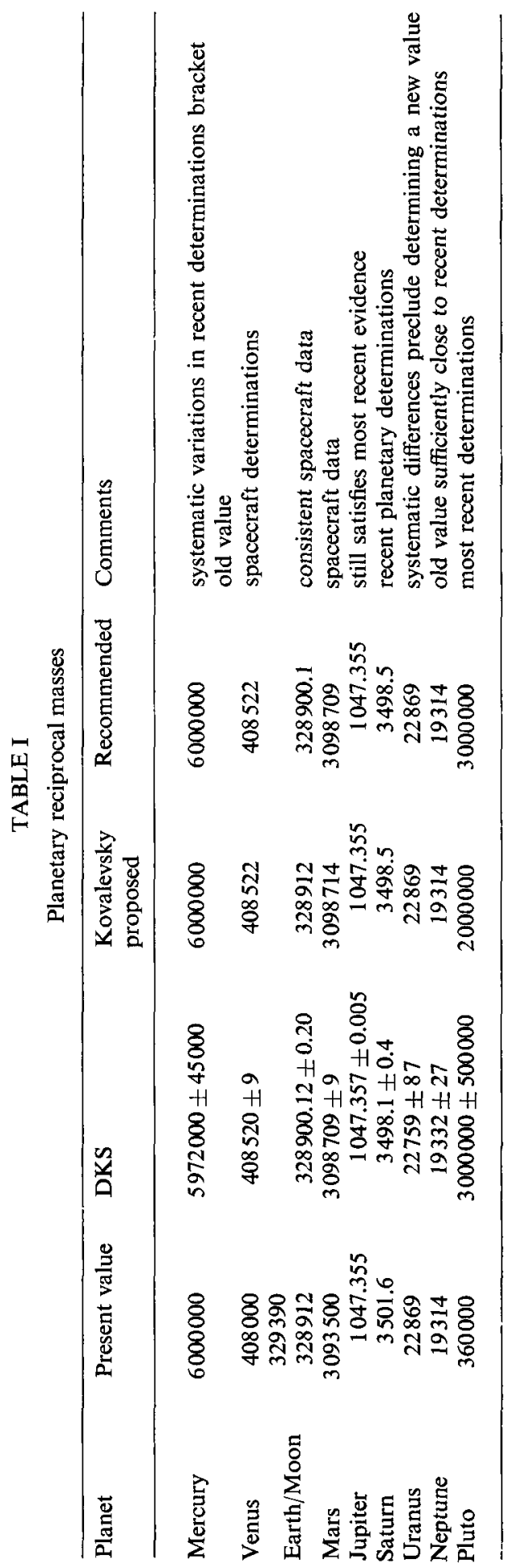


system of masses, constants and ephemerides, including changes to tentatively adopted constants, could be adopted at the 1976 IAU. This would permit adoption of new ephemerides in time for publication in the 1980 editions of the AE.

\section{References}

Brown, E. W.: 1919, Tables of the Motion of the Moon, New Haven.

Duncombe, R. L., Klepczynski, W. J., and Seidelmann, P. K.: 1973, Fundamentals of Cosmic Physics, Gordon and Breach, New York.

Eckert, W. J., Brouwer, D., and Clemence, G. M.: 1951, Astron. Papers Am. Ephem. XII.

Gaillot, A.: 1904, Mem. Obs. Paris 24, 172.

Gaillot, A.: 1910, Ann. Obs. Paris 28, A1.

Gaillot, A.: 1913, Ann. Obs. Paris 31, 105.

Kovalevsky, J.: 1971, Celes. Mech. 4, 213.

Leverrier, U. J. J.: 1858, Ann. Obs. Paris 4, 1.

Leverrier, U. J. J.: 1859, Ann. Obs. Paris 5, 1.

Leverrier, U. J. J.: 1861a, Ann. Obs. Paris 6, 1.

Leverrier, U. J. J.: 1861b, Ann. Obs. Paris 6, 185.

Leverrier, U. J. J.: 1876a, Ann. Obs. Paris 12, 1.

Leverrier, U. J. J.: 1876b, Ann. Obs. Paris 12, A1.

Leverrier, U. J. J.: 1877a, Ann. Obs. Paris 14, A1.

Leverrier, U. J. J.: 1877b, Ann. Obs. Paris 14, 1.

Newcomb, S.: 1898a, Astron. Papers Am. Ephem. VI.

Newcomb, S.: 1898b, Astron. Papers Am. Ephem. VII.

Ross, F. E.: 1912, Monthly Notices Roy. Astron. Soc. 72, 342.

Ross, F. E.: 1917, Astron. Papers Am. Ephem. IX, Part II.

\section{DISCUSSION}

Kovalevsky: As far as the mass of Pluto is concerned, I refer to I. I. Shapiro who states that almost any value can be derived from the observations available at present.

My second remark has to do with the mass of the Earth-Moon system. If a value of $1 / 328900.1$ is adopted and the 1964 value of $1 / 328912$ is changed, then, there will be other consequences on the already adopted IUGG system for the Earth, and this may be unwise.

Mulholland: It seems inconsistent to be considering seriously an improvement of one order of magnitude in precession and then decline to accept a possible two orders of magnitude improvement of the Earth mass.

Vicente: I should like to support the remark of our chairman about the value he proposed for the mass of the Earth, in spite that it is different from Duncombe's recommended value.

I should like to emphasize that our Union has to acknowledge the values of certain constants proposed by other unions. In this case, the International Union of Geodesy and Geophysics has adopted a set of conventional constants defining the Geodetic Reference System 1967: equatorial radius for the Earth $a_{e}$, geocentric gravitational constant of the Earth $G M$, dynamical form factor of the Earth $J_{2}$.

We have to accept the values proposed in the same way that other unions will have to accept the new system of astronomical constants we intend to set up in 1980. 